\title{
Foot Modeling and Smart Plantar Pressure Reconstruction from Three Sensors
}

\author{
Hussein Abou Ghaida, Serge Mottet and Jean-Marc Goujon*
}

CNRS-Foton, UMR 6082, Lannion, France

\begin{abstract}
In order to monitor pressure under feet, this study presents a biomechanical model of the human foot. The main elements of the foot that induce the plantar pressure distribution are described. Then the link between the forces applied at the ankle and the distribution of the plantar pressure is established. Assumptions are made by defining the concepts of a 3D internal foot shape, which can be extracted from the plantar pressure measurements, and a uniform elastic medium, which describes the soft tissues behaviour. In a second part, we show that just 3 discrete pressure sensors per foot are enough to generate real time plantar pressure cartographies in the standing position or during walking. Finally, the generated cartographies are compared with pressure cartographies issued from the F-SCAN system. The results show $0.01 \mathrm{daN}$ ( $2 \%$ of full scale) average error, in the standing position.
\end{abstract}

Keywords: 3D foot shape, biomechanics, foot modeling, numerical simulation, plantar pressure cartography, pressure sensors, instrumented insole.

\section{INTRODUCTION}

Foot modeling and plantar pressure measurements have widely focused interest of researchers. Plantar pressure measurements are used in the diagnostics of foot related problems such as plantar foot pain or diabetic foot [1-3]. In the quiet standing position or during walking, the plantar pressures highlight the compression of the soft tissues (skin, fat, ligaments, and muscles) under the feet. The plantar pressure distribution can be measured by using insoles made of a pressure sensor matrix. F-SCAN ${ }^{\circledR}$ mobile System [from Teckscan, Inc. Boston, MA. 02127-1309, USA] with 954 sensors or Pedar ${ }^{\circledR}$ with 256 sensors [from Novel, Gmbh novel GmbH, Ismaninger Str. 51, D-81675 Munich] are the most popular instrumented insole systems used by podiatrists. Information processed from the plantar pressure distributions are used to design a special footwear or insole. This footwear (or foot insole) inserted between the foot and the ground may help in the treatment and prevention of foot pathology [4-6]. The design of the appropriate footwear by the podiatrist is generally based on pressure measurements. This kind of design is rather empirical and needs simulation tools to predict the plantar pressure cartography as a function of the thickness, stiffness and shape of the footwear.

Foot modeling may target different applications: prediction of plantar pressure, behaviour during balance, prevention of ulcer formation, gait or running analyses, etc.

*Address correspondence to the author at the Laboratoire Foton, ENSSAT, CS 80518, 6 rue Kerampont, 22305 Lannion Cedex, France;

Tel: +332964690 52; ; Fax: +33296469076;

E-mail: Jean-Marc.Goujon@enssat.fr
Many researchers focus on the biomechanical foot model. One can mention 3D finite element models of the foot and ankle, which have been developed to study ulcer formation $[7,8]$, or to predict the forces between the foot and different types of support, such as moulded insoles, insoles of varying stiffness [9-12]. Models of coupled foot-boot [13] have also been proposed.

This modeling technique requires prior knowledge of the foot shape in 3D, provided by Magnetic Resonance Imaging (MRI) measurements, Computed Tomography (CT) scanners or video reconstruction [14]. Unfortunately, most podiatrists do not have these expensive and sophisticated devices at their disposal. With the aim of offering the podiatrist a practical tool able to simulate plantar pressure distribution, we have derived a new model of the foot, taking into account the internal foot shape and position, which is suitable for dynamic balance description. The model must also take into account the properties of the shoe insole. Of course this model will have to work effectively with only the data that podiatrists can measure on their own.

The objective is to provide a model generating the plantar pressure cartography for different foot positions (seat) and different elastic media under the foot. At first, the model of a bare foot on planar ground is presented. The extraction of the foot parameters from the plantar pressure cartographies is detailed in the standing position. The results show the extrapolation of the plantar pressure cartography when using three pressure sensors. In the second part, the case of a foot in a shoe is presented and the results during walking are shown. 


\section{METHODS}

\section{Foot Model and Hypothesis}

In the standing position, equilibrium is controlled by the muscle action, which moves the distribution of the plantar pressure by changing the rotation of the foot around the ankle (anterior-posterior) and the repartition of the total weight of the body on both feet (lateral).

The force applied to the ankle compresses the foot, which can be considered foremost as a skeleton covered by an elastic medium made of soft tissues. The compression naturally increases plantar pressure.

One can consider that the foot skeleton can be divided into three parts, from back to front: the heel, the metatarsus and the phalanges. The soft tissues of the plant constitute a complex medium. The thickness and elasticity of the soft tissues depend on their location under the foot, but the heel and the metatarsal heads support the biggest pressure.

A simplified model has been developed in order to describe the relationship between posture and plantar pressure through mechanical foot characteristics.

In standing position, at rest, the sum of the forces perceived on the surface of the two feet corresponds to the force resulting from the total body mass under the acceleration of gravity. When walking, the force resulting from the vertical acceleration of the centre of mass has to be added.

\section{Bare Foot on Planar Ground}

At first, one considers the case of a bare foot on a planar ground.

In order to describe the relationship between the force applied to the ankle and the plantar pressure distribution, we can define three elements for the mathematical model: FS, the foot seat, IS the internal foot shape, and EM the elastic medium. The parameters of each element are foot dependent.

\section{Foot Seat}

The origin of the internal coordinates of the foot is the ankle, $x$ being the lateral coordinate axis, $y$ the longitudinal one (heel to forefoot) and $z$ the vertical one.

The location of the foot in space is given by the vertical position of the ankle and the rotations around the ankle, as shown in Fig. (1). The equation of the "foot seat" FS plan is:

$$
z_{F S}(x, y)=\tan (\alpha) \cdot x+\tan (\beta) \cdot y+c=a \cdot x+b \cdot y+c
$$

The terms $a \cdot x$ and $b \cdot y$ respectively describe the lateral and longitudinal rotations around the ankle, whereas $c$ is the vertical displacement of the ankle.

The considered rotation angles are small. In the approximation of small angles $a=\tan (\alpha)=\alpha \quad$ and $b=\tan (\beta)=\beta$

\section{Internal Foot Shape}

The Internal foot Shape, IS, is a surface profile describing the non-compressive material mainly due to the skeleton.

The internal foot shape is described by the distance of each foot point to the foot seat plane. Since the rotation angles are small, the distance has its main component, $z_{I S}(x, y)$, along the $z$ axis. The components along the $x$ and $y$ axes due to the rotations are neglected.

IS can be precisely obtained by "classical" 3D measurement techniques, such as MRI or scanning. But it can also be approached by simple plantar pressure measurements.

For a bare foot on flat ground, the foot shape is similar to a 3D profile of the underfoot, such as the one provided by a 3D scanner. The internal foot shape consists in the foot skeleton covered by the other rigid media, such as the ligaments and the muscles, as shown in Figs. ( $\mathbf{2 A}$ and $\mathbf{2 B}$ ).

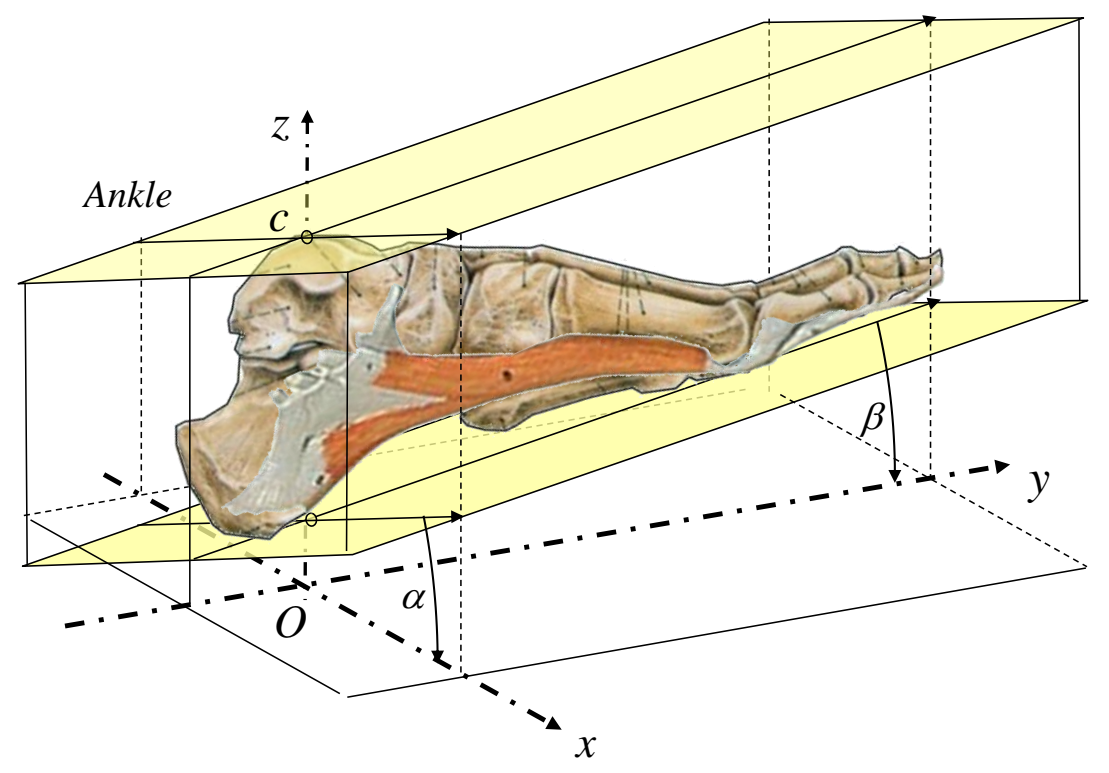

Fig. (1). Illustration of the foot seat. 


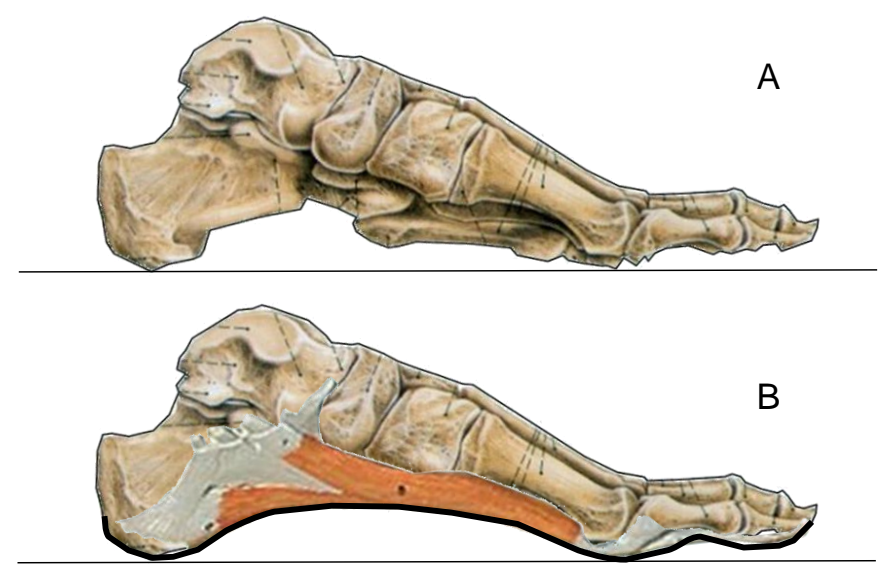

Fig. (2). Foot model. A) foot skeleton, B) internal foot shape.

\section{Elastic Medium}

The internal foot shape (skeleton and rigid medium) is covered by soft tissues, as shown in Fig. (3C). The thickness and the elasticity of the soft tissues depends on the underfoot location. The compression of the tissues induces a pressure.

To simplify the modeling, it is assumed that the soft tissues can be described realistically by an equivalent uniform elastic medium (EM). The elastic medium is uniform in terms of thickness and elastic properties, Fig. (3D). For a bare foot, the Elastic Medium, EM, describes directly the soft tissues, which cover the internal foot shape.

The relation between pressure and compression of the EM should verify the following properties:

- The EM does not apply any force when the foot is not in contact with the floor.

- The EM cannot be compressed beyond its thickness.

- The intensity of the force depends on the material stiffness.

- For the same compression, the force depends on thickness and stiffness of the EM.

The vertical compression of the EM is directly related to the FS and IS functions. If there is a contact, that is to say if

$$
z_{C}(x, y) \geq 0 \text {, then } z_{C}(x, y)=z_{F S}(x, y)+z_{I S}(x, y) \text {. }
$$

The usual linear spring compression models, issued from the small variation hypothesis, cannot be applied in this case. A simple function that can account for the above limitations is an exponential function, which links the EM compression to the applied force depending on the characteristics of the EM. It is given by:

$$
\begin{aligned}
& z_{C}=\text { thick } \cdot\left(1-\exp \left(-\frac{F}{\text { stiff }}\right)\right) \text { or the inverse function } \\
& F=- \text { stiff } \cdot \ln \left(1-\frac{z_{C}}{\text { thick }}\right) \text { for } F \geq 0 \text { or } 0 \leq \frac{z_{C}}{\text { thick }}<1 \text { where } z_{C}
\end{aligned}
$$

(mm) is the compression of the EM, thick (mm) the thickness, stiff $\left(\mathrm{N} / \mathrm{cm}^{2}\right)$ the stiffness, and $F\left(\mathrm{~N} / \mathrm{cm}^{2}\right)$ the pressure.

This type of law can be verified through the experimental compression curve of an $8 \mathrm{~mm}$ thick elastomer foam with a $25 \mathrm{~cm}^{2}$ area is shown on Fig. (4). The dots are the measured points, the line is the fitted exponential with a stiffness of 0.2 $\mathrm{daN} / 25 \mathrm{~cm}^{2}$. This type of material is used for insoles.

The $25 \mathrm{~cm}^{2}$ area compares with the minimum support surface of the foot, when standing on the forefoot or the heel.

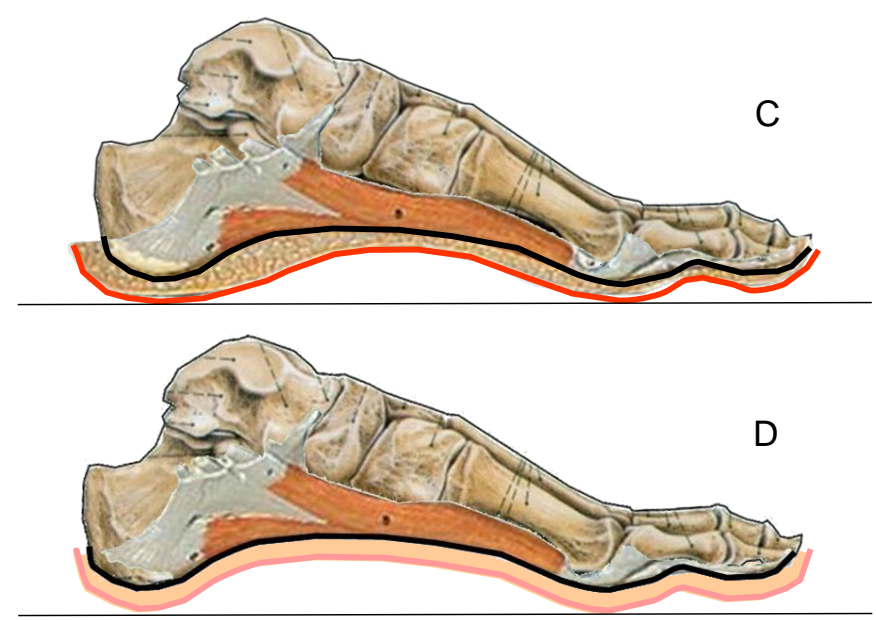

Fig. (3). Elastic medium: C) in red, soft tissues, D) in pink, modelled elastic medium. 


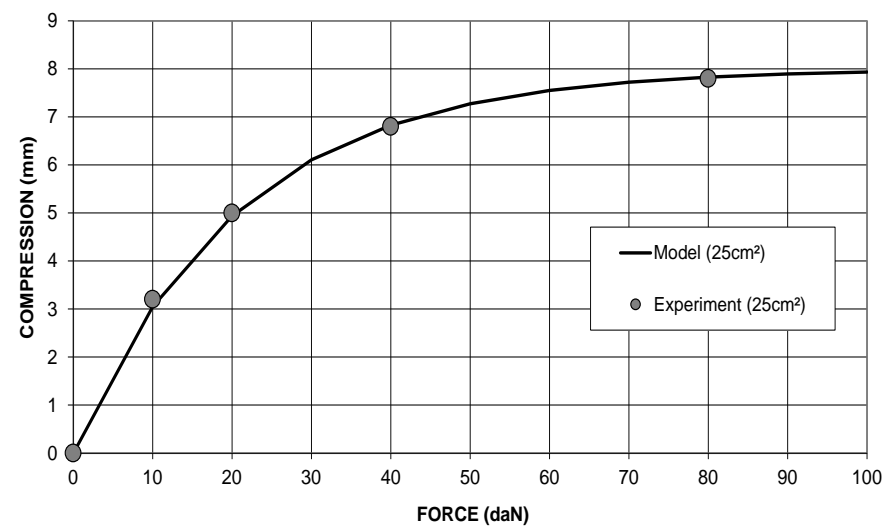

Fig. (4). Experimental validation of exponential behaviour of an elastomer medium.

\section{Pressure Map}

The problem is spatially discretised. The size of a pixel corresponds to the F-SCAN one, $S_{p}=0.508 \times 0.508 \mathrm{~cm}^{2}$, which is being used for the experiments.

The pressure cartography, $F\left(z_{F S}\right)$, is directly derived from the foot seat $z_{F S}(x, y)$, for a given internal foot shape $z_{I S}(x, y)$ and a given elastic medium. With, stiff in daN per pixel, the force per pixel is expressed by: $F(x, y)=-$ stiff $\cdot \ln \left(1-\frac{z_{C}(x, y)}{\text { thick }}\right)$ with $z_{C}(x, y)=\max \left(z_{F S}(x, y)+z_{I S}(x, y), 0\right)$

Direct simulation of the plantar pressure distribution as a function of the foot seat FS can be achieved with arbitrary IS and EM, but for medical applications, the simulation requires the actual internal foot shape of the patient and the elastic medium characteristics. data.

Our purpose is to identify FS and EM from experimental

\section{Internal Foot Shape Extraction}

The pressure measurements are made with F-SCAN, at a frequency of 50 scans per second.

From these measurements one can obtain a set of pressure maps for different foot seats.

At each time step, $i=1 \ldots M$, for each pixel $k=1 \ldots N$ of the foot the force $F_{i}^{k}$ is measured and stored. Note that each time step corresponds to a different foot seat.

$F_{i}^{k}$ and the compression $z_{C_{i}}{ }^{k}$ are linked by the bijective relation: $z_{C i}{ }^{k}=$ thick $\cdot\left(1-\exp \left(-\frac{F_{i}^{k}}{\text { stiff }}\right)\right)$

The compression $z_{C i}^{k}$ depends on the seat plan $z_{F S}{ }_{i}^{k}=a_{i} \cdot x_{k}+b_{i} \cdot y_{k}+c_{i}$ and the internal foot shape $z_{I S k}$ : $z_{C i}^{k}=a_{i} \cdot x_{k}+b_{i} \cdot y_{k}+c_{i}+z_{I S}{ }^{k}$
The relation can be written:

$\frac{a_{i}}{\text { thick }} \cdot x_{k}+\frac{b_{i}}{\text { thick }} \cdot y_{k}+\frac{c_{i}}{\text { thick }}+\frac{z_{I S}{ }^{k}}{\text { thick }}=\left(1-\exp \left(-\frac{F_{i}^{k}}{\text { stiff }}\right)\right)$

The unknowns are the thickness, thick, the stiffness, stiff, the 3 foot seat parameters $\left(a_{i} ; b_{i} ; c_{i}\right)$ for each step $i$ and the internal foot shape $z_{I S}{ }^{k}$ for each pixel $k$. But the relation shows that the thickness thick appears as a proportional factor for the other unknown spatial parameters $\left(a_{i} ; b_{i} ; c_{i} ; z_{I S}{ }^{k}\right)$. There is no other relation, so that the thickness cannot be determined independently of $\left(a_{i} ; b_{i} ; c_{i} ; z_{I S}{ }^{k}\right)$. It is fixed to an arbitrary realistic value acting as a proportional factor on the foot seat and the internal foot shape.

For $k=1 \ldots N$ the foot seat parameters $a_{i} ; b_{i} ; c_{i}$ are unknown, along with the profile $z_{I S}$ for $k=1 \ldots N$.

The number of data $z_{C}{ }^{k}$ is equal to $N \times M$. The number of unknown parameters are the $N$ values of $z_{I S}{ }^{k}$, and the $3 \times M$ values of the foot seat parameters $\left(a_{i} ; b_{i} ; c_{i}\right)$.

The total number of data $N \times M$, is larger than the total unknown values $N+3 \times M$.

After eliminating the unnecessary pixels, $N$ is of the order of 500, and $M$ is of the order of 1000 or more, depending on the duration of the measurement.

Most of the unknown parameters can be obtained by a linear system, which minimizes the estimation error.

Let be $E$ the summation of the square of the errors made on the compression, which can be written:

$$
E=\sum_{i=1}^{M} \sum_{k=1}^{N}\left(\text { thick } \cdot\left(1-\exp \left(-\frac{F_{i}^{k}}{\text { stiff }}\right)\right)-\left(a_{i} \cdot x_{k}+b_{i} \cdot y_{k}+c_{i}+z_{I S}{ }^{k}\right)^{2}\right.
$$

where the $F_{i}^{k}$ are the experimental data. 
The thickness is fixed. The values of the other unknowns can be computed by expressing that the partial derivatives of $E$ with respect to each unknown are null at the minimum of $E$ (least squares method).

$$
\begin{aligned}
& \forall i: \frac{\partial E}{\partial a_{i}}=0 ; \frac{\partial E}{\partial b_{i}}=0 ; \frac{\partial E}{\partial c_{i}}=0 ; \\
& \forall k: \frac{\partial E}{\partial z_{I S_{k}}}=0 ; \frac{\partial E}{\partial \text { stiff }}=0 .
\end{aligned}
$$

The partial derivatives, $\frac{\partial E}{\partial a_{i}}=\frac{\partial E}{\partial b_{i}}=\frac{\partial E}{\partial c_{i}}=\frac{\partial E}{\partial z_{I S_{k}}}=0$, lead to a linear equation system in $\left(a_{i} ; b_{i} ; c_{i} ; z_{I S}{ }^{k}\right)$. But, $\frac{\partial E}{\partial s t i f f}=0$ do not lead to a linear form. A first order development could have been used. We preferred to compute the stiffness which minimises $E$ with method close to a dichotomy method. In the present case, the variation domain of the unknown is divided in 3 parts instead of 2. At first, the domain of variation of the stiffness is divided in 3 intervals, which correspond to 4 values of the stiffness. For each value of the stiffness, the least squares method is used to determine the $a_{i} ; b_{i} ; c_{i} ; z_{I S}{ }^{k}$ parameters. The $E$ value is then computed. The two neighbour values surrounding the stiffness, for which the $E$ value is the lowest, define the new boundaries of the variation domain, which is in turn divided in 3 intervals. This iterative process is repeated until the required accuracy of the stiffness is reached.

The numerical method has been developed with MATLAB software on an ordinary desktop personal computer. The computing time is about 10 seconds.

In the following, we present a measurement of the plantar pressure distribution, on a $70 \mathrm{~kg}$ male subject with a foot length of $26 \mathrm{~cm}$, bare-footed, in a standing position. The F-

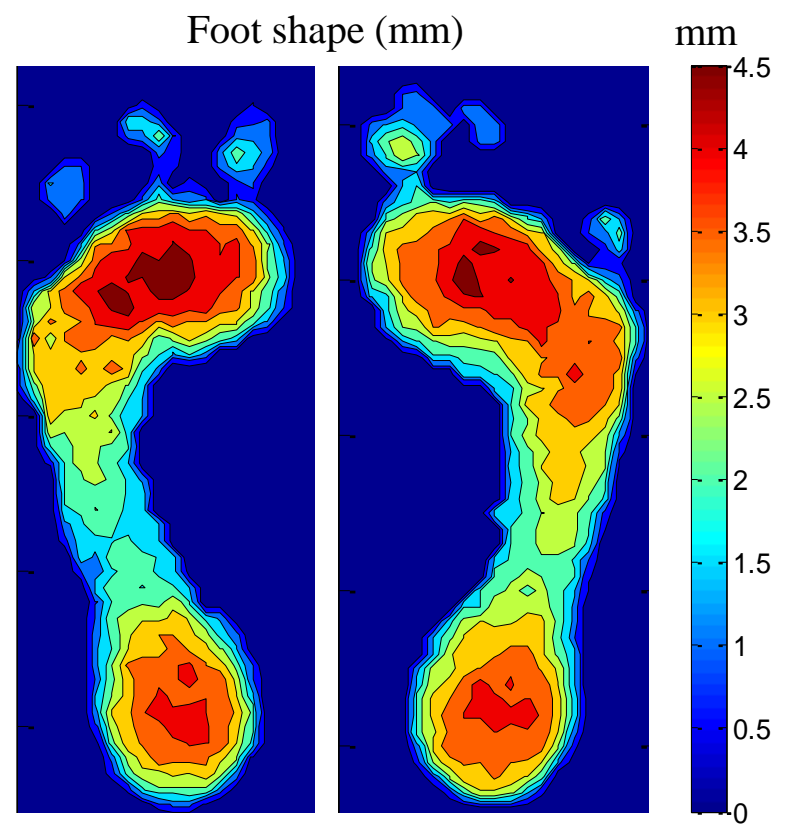

Fig. (5). Internal foot shape altitude in $\mathrm{mm}$.
SCAN pressure maps are recorded at a frequency of 50 frames/second for 1 minute.

The thickness of the elastic medium has been fixed at $5 \mathrm{~mm}$, a realistic value that corresponds to the mean footpad thickness under the heel and the forefoot. The calculated internal foot shape is shown in Fig. (5). The height varies between 1 and $4.5 \mathrm{~mm}$. A variation of $2 \mathrm{~mm}$ can be observed between the mid-foot and the forefoot or the heel.

Fig. (6) presents the standard deviation for each pixel $k$, around the average value computed from the different postures $i$. The amplitude is less than $0.15 \mathrm{~mm}$ for the main support zones. The standard deviation is the result of implicit errors due to the simplified model hypothesis and measurement uncertainty.

The low values of standard deviation, compared to the height of Internal Shape, validate the hypothesis and the model. Higher variation amplitude points are located on the toe phalanges, which are articulated with the metatarsus and may move independently to the foot seat.

Other high variation points may appear in the zones where only a few pressure data are available. In any case the precision is less than a few percent.

\section{SUMMARY}

After identification of the parameters for each pixel $k$ at each step $i$ the seat and the compression of the elastic medium are known together with the internal foot shape, which is independent of the step.

Then it is possible to calculate $\tilde{F}_{i}^{k}$, the force applied to the pixel $\mathrm{k}$ for any seat $z_{F S_{i}}$ defined by $\left(a_{i}, b_{i}, c_{i}\right)$

$$
\tilde{F}_{i}^{k}=-s t i f f \cdot \ln \left(1-\frac{z_{C_{i}}^{k}}{\text { thick }}\right)
$$

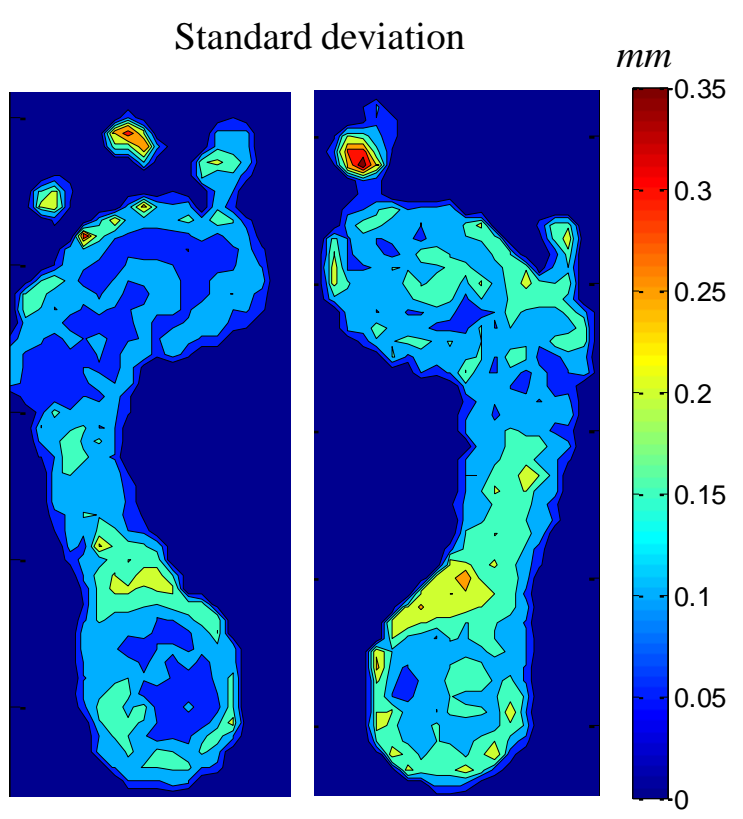

Fig. (6). Internal foot shape standard deviation in $\mathrm{mm}$. 
with $z_{C_{i}}{ }^{k}=z_{F S}{ }^{k}+z_{I S}{ }^{k}=a_{i} \cdot x_{k}+b_{i} \cdot y_{k}+c_{i}+z_{I S}{ }^{k}$

\section{RESULTS}

\section{Plantar Pressure Extrapolation from 3 Sensors}

Since the foot seat is defined by the equation of a plane, three sensors are sufficient to measure it.

The concept has first been tested using an arbitrary IS and fixed EM, varying the foot seat in order to obtain the necessary database. A study has been carried out to evaluate the performances of the method independently of experimental measurement incertitude.

It allowed us to verify that the use of 4 or 5 sensors rather than 3 did not significantly change the precision of the results.

The 3 sensors are fixed on an insole. They are arranged under the heel, the first and fifth metatarsal respectively, as shown in Fig. (7). The F-SCAN insole is placed above the sensor insole, in order to compare the plantar pressures and the sensor measurement.

\section{Sensor Parameter Extraction}

The effective stiffness value for the sensors has to be determined. The stiffness depends on the surface of the sensor. Presently, the actual surface and response of the sensors are the stiffness value.

The internal foot shape and the seat have been determined for each step $i$. The value of the stiffness of the sensors is calculated using the same method as EM stiffness with the same thickness and from the same data base.

Moreover the exact positions of the sensors with regard to the F-SCAN insole are unknown. Practically the two insoles have no reason to correspond perfectly.

The relative distances of the 3 sensors from each other, on the insole, are known. The shift (rotation and translation)
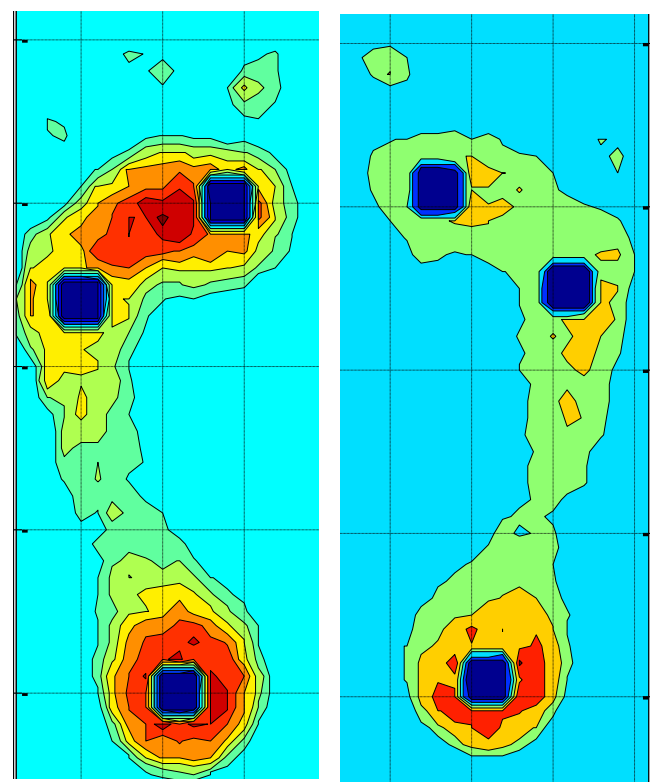

Fig. (7). Sensor positions under the left and right foot. of the sensor coordinate, with regard to the F-SCAN one, is numerically determined simultaneously along with the extraction of the stiffness.

\section{Foot Seat Extraction from 3 Sensors}

At each time step $i$, the force measured by the sensors provides the compression of the elastic material above the sensors. Once the foot shape above each sensor $j=1 . .3$ is known, the foot seat extraction can be resumed by solving the equations to obtain the 3 unknowns $a_{i}, b_{i}$ and $c_{i}$.

$$
z_{C i}^{j}-z_{I S}=a_{i} \cdot x_{j}+b_{i} \cdot y_{j}+c_{i}
$$

After extracting the FS, and knowing the IS, the plantar pressure distribution map can be calculated.
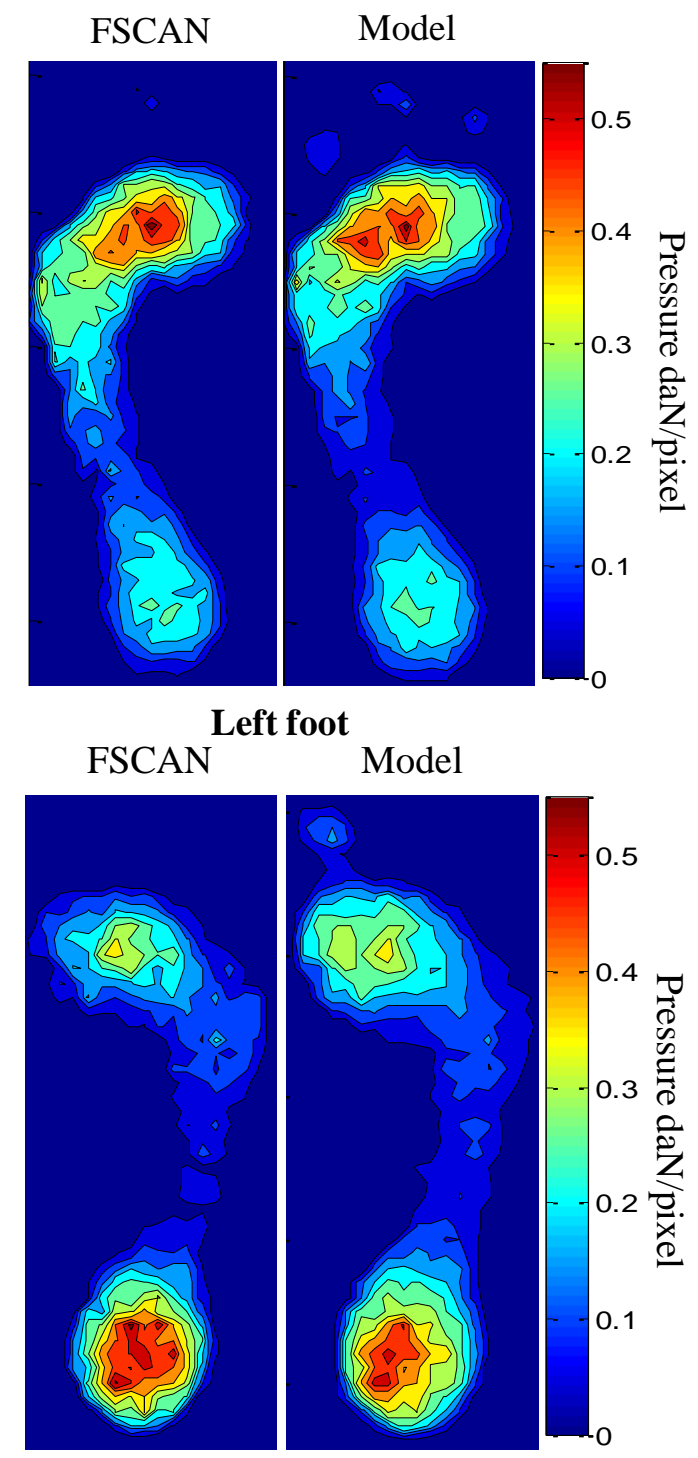

Right foot

Fig. (8). Left and right, foot plantar pressure measured and extrapolated during standing. 


\section{Plantar Pressure Simulation}

For each posture, the foot seat is determined from the 3 sensors responses. Using the foot seat, the calculated foot shape and the stiffness of the elastic medium the pressure maps are then real time simulated.

We now compare the simulated maps to the measured FScan pressure maps. Fig. (8) shows the plantar pressure measured for one of the standing positions that the extrapolated plantar pressure from the model highlights the anatomically support zones under the foot, such as the heel and the forefoot.

One can notice that the peak pressure value under the heel for the left and right foot is about $0.45 \mathrm{daN} /($ pixel area) for both measured and extrapolated pressures. We see that the pressure under the toes does not appear in this posture.

The average error measured at each pixel is $0.01 \mathrm{daN}$, with a standard deviation of $0.005 \mathrm{daN}$. This model therefore offers good prediction characteristics. It will be verified on a wider sample of subjects in further studies.

\section{Foot in a Shoe}

Previously we were considering a bare foot. The elastic medium EM was the plantar soft tissues.

If the foot lies on an insole, the EM is composed of several layers of different thicknesses and stiffnesses. Usually, the stiffness of soft tissues and insoles may be comparable. When identifying the stiffness of the EM from pressures data it is impossible to separate the contributions of the soft tissues and the insole.

In this case, we show in Fig. (9) that a single exponential satisfies the description of a multilayer system for the domain of pressure between 0.15 and 1 daN/pixel, which corresponds to the pressure of greater numerical weight in the linear regression method. It is impossible to determine the zero value of the functions, which implies that the compression can be defined with an additive constant. For example, Fig. (9) shows the summation, in circular forms, of two $5 \mathrm{~mm}$ layers with respective stiffness of $0.1 \mathrm{daN} /$ pixel and $0.4 \mathrm{daN} /$ pixel. The continuous black curve is the fitting exponential of the sum for a thickness of $7.6 \mathrm{~mm}$ and a stiffness of $0.25 \mathrm{daN} /$ pixel.

So the elastic material is considered as a mean equivalent medium describing the multilayer behaviour in the main domain of pressures. The model and the method are still valid.

When the foot is inside a shoe, the shape of the shoe insole changes the problem. In such a case the compression is the distance between the internal foot shape and the shoe insole.

$$
z_{C}=z_{F S}(x, y)+z_{I S}(x, y)+z_{\text {Shoe }}
$$

The shape of the shoe insole need not be known. The shoe shape will be included in the internal foot shape when identifying the parameters.

$$
z_{C}=z_{F S}(x, y)+\hat{z}_{I S}(x, y)
$$

Where the new internal foot shape function is

$$
\hat{z}_{I S}(x, y)=z_{I S}(x, y)+z_{\text {Shoe }}
$$

The internal foot shape has to be considered as a numerical function, which may look different from a bare foot shape. But it does not change the capability of the method to extrapolate the foot pressures from 3 pressure sensors.

For illustration purposes, Fig. (10) presents a comparison between measured and simulated plantar pressure maps during walking for the same subject.

The simulated map for the left foot describes the plantar pressure evolution during heel strike, mid stance, forefoot.

\section{DISCUSSION}

The extrapolations of the plantar pressure maps, from 3 sensors per foot, are made by using the foot model developed in this study. This method depends on the foot parameters. For a bare foot on a planar ground, the IS and the stiffness of the EM are determined from the plantar pressure maps issued from F-Scan.

The extraction of the IS from the plantar pressure maps while the subject is standing, exhibits a variance about a few

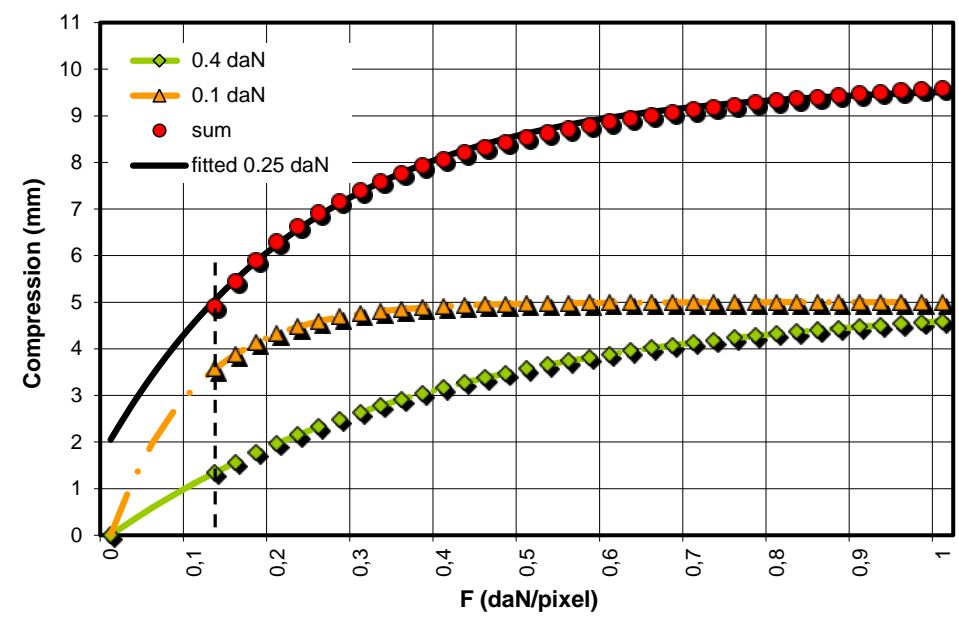

Fig. (9). Approximation of a multi exponential problem using a single exponential function. 


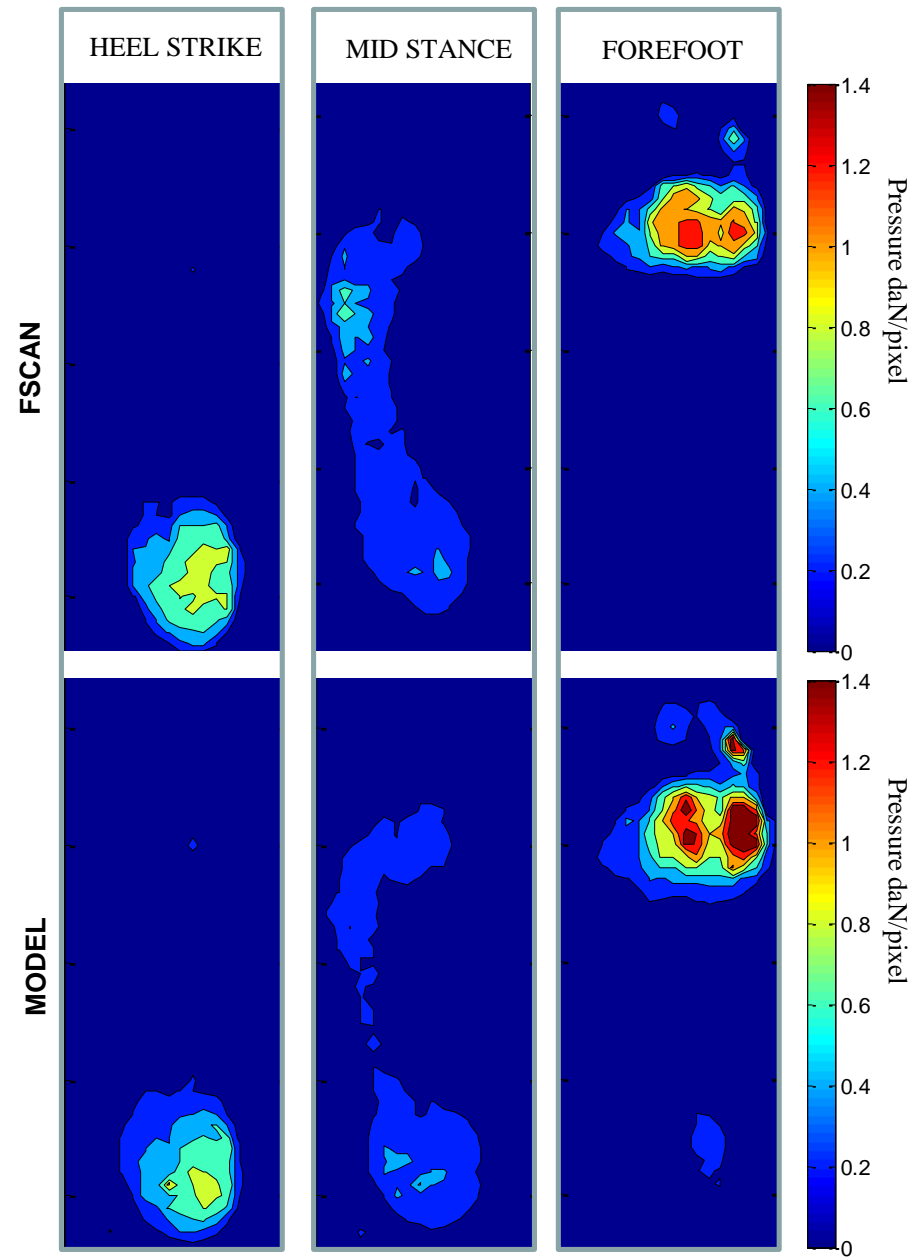

Fig. (10). Left foot plantar pressure cartography during walking.

percent under the heel and the mid foot. A more important standard deviation (12\% of the measured height) can be seen under the toes. This variation is due to the relatively low pressure measured under the toes during calibration. A possible solution would be the application of a specific pressure under the toes when making measurements.

Starting from these barefoot measurements, the height of the extracted foot shape is extracted for a selected thickness of $5 \mathrm{~mm}$. This value is comparable to the thickness of the plantar soft tissue. Otherwise, when the patient is standing on an elastomeric insole during measurements, the total thickness value has to be adjusted, depending on the stiffness of each medium and mechanical combination law.

For a foot in shoe, it should be noted that using a compensated insole during the measure of the plantar pressure changes the IS. In this case, the calculated IS include the form of the compensated insoles and the IS calculated when bare foot.

Thanks to the foot model developed in this study, the plantar pressure maps are calculated from the measures of 3 pressure sensors per foot. In the standing position, the average difference on the force map per pixel, compared to F-Scan one is $0.01 \mathrm{daN} / \mathrm{Pixel}$, for a pressure variation between 0 and $0.45 \mathrm{daN} / \mathrm{Pixel}$.
We also verified the validity of the method during walking. The plantar pressures maps have been also extrapolated for the same subject. Results show a high similarity in the pressure maps compared to F-Scan one. Therefore, the plantar pressure extrapolated during walking permits studies on the gait.

Moreover, the results confirm the good approximation of a uniform elastic medium with exponential elasticity properties. This assumption limits the complexity of modeling the plantar soft tissues, and foot insoles.

For simplicity, the model sets that the IS remains rigid when changing the FS. But as the toe phalanges are articulated to the metatarsus, they may move differently from the FS. Some error may occur on the under toe pressures, but they remain small with regards to heel or forefoot pressures.

The results confirm the hypotheses suggested, simplifying the foot model.

Note that the model validation has been carried out on a healthy foot, with "standard" pressure areas. Specific sensor number, location and size may be necessary in other cases. The model aims to provide simplified practical tool to generate pressure maps, inducing a cost/benefits balance concerning sensor number and complexity. 


\section{CONCLUSION}

We have proposed a new foot model based on simplified anatomical modeling. It provides a practical tool to simulate the plantar pressure distribution for podiatrists.

Based on the foot model, the plantar pressure distribution can be generated using only three pressure sensors per foot. For this, the internal foot shape is first calculated from plantar pressure cartographies, issued from usual matrix systems, such as F-SCAN or Pedar, avoiding the use of expensive equipment.

The sensors are to be used in a portable ambulatory monitoring system, with low power consumption and wireless transmission (Wi-Fi, Bluetooth...). The reduced number of data would be transmitted to podiatrist, or locally processed within a smart phone processor. Plantar pressures cartographies simulations are real time proceeded.

This system could be suitable for the balance study for the elderly or preventing ulcers formation in diabetic foot. These applications will be presented elsewhere.

In the future, this model could be further developed to extrapolate the action of added insoles on the under foot pressure when assigning thickness and stiffness to each area of the elastic medium. It could be an insole design tool for diabetic patients.

\section{CONFLICT OF INTEREST}

The authors declare that there is no conflict of interest associated with this work.

\section{ACKNOWLEDGEMENTS}

This work is supported by a grant from French Research Ministry and from Lannion Trégor Agglomération, Région Bretagne and Conseil Général des Côtes d'Armor through the PONANT project. The authors would like to acknowledge N. Le Berre (Lannion Institute of Tech.), for experimental measurement on the elastomeric, Dr D. Baron (Trestel Hospital, rehabilitation \& rheumatology), J. Durrmann (Durrmann SA, podiatry) for the F-Scan system and helpful discussions, D. Bosc and A. OHare for help in correcting and submitting this manuscript.

\section{ABBREVIATIONS}

$\begin{array}{lll}\mathrm{CT} & = & \text { Computed Tomography } \\ \mathrm{EM} & = & \text { Elastic Medium }\end{array}$
FS
$=$
Foot Seat
IS $=$
Internal foot Shape
MRI
Magnetic Resonance Imaging

\section{REFERENCES}

[1] A. Amemiya, and H. Noguchi, "Relationship between elevated plantar pressure of toes and forefoot and gait features in diabetic patients", 35th Annual International Conference of the IEEE EMBS Engineering in Medicine and Biology Society, Osaka, Japan, vol. 2, pp. 4633-4636, 2013.

[2] N.L.W. Keijsers, N.M. Stolwijk, J. W. K. Louwerens, and J. Duysens, "Classification of forefoot pain base on plantar pressure measurements", Clinical Biomechanics, vol. 28, no. 3, pp. 350-356, 2013.

[3] W.R. Ledoux, J.B. Shofer, M.S. Cowley, J.H. Ahroni, V. Cohen, and E.J. Boyko, "Diabetic foot ulcer incidence in relation to plantar pressure magnitude and measurement location", Journal of diabetes and its complications, vol. 27, pp. 621-626, 2013.

[4] S.A. Bus, J.S. Ulbrecht, and P.R.Cavanagh, "Pressure relief and load redistribution by custom-made insoles", Clinical Biomechanics, vol. 19, pp. 629-638, 2004.

[5] G. Luo, V.L. Houston, M.A. Garbarini, A.C. Beattie, and C. Thongpop, "Finite element analysis of heel pad with insoles", Journal of Biomechanics, vol. 44, no. 8, pp. 1559-1565, 2011.

[6] J. Paton, B. Graham, J. Ray, and E. Stenhouse, "Effectiveness of insoles used for the prevention of ulceration in the neuropathic diabetic foot: a systematic review", Journal of Diabetes and Its Complications, vol. 25, pp. 52-62, 2011.

[7] V. Luboz, M. Bucki, I. Stavness, F. Cannard, and Y. Payan, "Biomechanical Modeling of the Foot To Study and Prevent the Formation of Ulcers", Journal of Biomechanics, vol. 45 no. S1, pp. S192, 2012.

[8] J.-C. Teoh, T. Lee, C.H. Lim and J.-H. Low , "Influence of Gastrocnemius-Soleus Muscle Force on Sub-Mth Load Distribution”, Journal of Biomechanics, vol. 45, no. S1, pp. S194, 2012.

[9] Chen, W.-P., Ju, C.-W., Tang, F.-T., "Effects of total contact insoles on the plantar stress redistribution: a finite element analysis", Clinical Biomechanics, vol. 18, no. 6, pp. S17-S24, 2003.

[10] J.T.-M. Cheung, and B.M. Nigg, "Clinical Applications of Computational Simulation of Foot and Ankle", Sports Orthopaedics and Traumatology, vol. 23, no. 4, pp. 264-271, 2008.

[11] J.T.-M. Cheung, and M. Zhang, "A 3-dimensional finite element model of the human foot and ankle for insole design", Archives of physical medicine and rehabilitation, vol. 86, no. 2, pp. 353-358, 2005.

[12] J.T.-M. Cheung, M. Zhang, A.K.-L. Leung, and Y.-B Fan, “Threedimensional finite element analysis of the foot during standing - a material sensitivity study", Journal of Biomechanics, vol. 38, no. 5, pp. 1045-1054, 2005

[13] T.-X.Qiu, E.C. Teo, Y.B. Yan, and W. Lei, "Finite element modeling of a 3D coupled foot-boot model" Medical Engineering \& Physics, vol. 33, no. 10, pp. 1228-33, 2011.

[14] A.K. Thabet, E. Trucco, J. Salvi, W. Wang, and R.J. Abboud, "A dynamic 3D foot reconstruction system", Annual International Conference of the IEEE Engineering in Medicine and Biology Society, 2011, pp. 599-602.

This is an open access article licensed under the terms of the Creative Commons Attribution Non-Commercial License (http://creativecommons.org/licenses/by-nc/3.0/) which permits unrestricted, non-commercial use, distribution and reproduction in any medium, provided the work is properly cited. 